\title{
Ecological, Economical and Technological Aspects of Development. Decomposition Analysis of Energy Consumption Related to $\mathrm{CO}_{2}$ Emissions in Ecuador
}

\author{
Andrés Robalino-López ${ }^{1}$, Zanna Aniscenko² \\ Escuela Politécnica Nacional, Quito-Ecuador, andres.robalino@epn.edu.ec ${ }^{I}$,University of Seville, Seville- \\ Spain,zanna.aniscenko@gmail.com²
}

\begin{abstract}
Decomposition Analysis (DA) is widely applied in understanding changes of economical, technological, environmental, and different indicators as energy consumption, employment and other socio-economic indicators. This work discusses the DA methodology and is applied within Ecuadorian case. We present the used technique, the applied mathematical methodology and the construction of an appropriate identity to measure the change of $\mathrm{CO}_{2}$ emission in Ecuador during the period of 1980-2025. Change is measured in both macro and disaggregated sectorial level. Specific aspects related to the application of DA to both the historical period (1980-2010) and in medium term prevision (20112025) for four proposed macro scenarios are discussed. The findings show that the evolution of the BS scenario, which implies a trend-growth GDP scenario, is almost a flat curve, however the $\mathrm{CO}_{2}$ emission increases steadily because of the absence of attenuation measurements. A similar behavior, although slightly sloping down, is observed for SC-2 scenario, where a rapid growth of the GDP is assumed without any attenuation action regarding to $\mathrm{CO}_{2}$ emissions. The other two scenarios, $S C-3$ and $S C-4$ show a steady reduction of the Dtot $=$ Dact ratio due to the changes in the sectorial structure and in the energy mix, which allows compensation of rapid GDP growth. This analysis suggests that, with the appropriate changes in the energy mix, the sectorial structure, and the share of renewable energies, Ecuador can move into a more environmentally sustainable state.
\end{abstract}

Keywords: Decomposition Analysis, Sustainable development, Ecuador.

\section{INTRODUCTION}

It is well known that humans have dramatically altered the global environment, but there is a limited understanding of the driving forces of these impacts. The absence of a refined set of analysis tools is cited as a fundamental limitation [1]. Analysis methodologies and tools have been developed in the field of analysis of decomposition, including sustainability framework known as the IPAT ${ }^{1}$ [2], [3]. The decomposition of changes in an aggregate environmental impact and of its driving forces has become popular to unravel the relationship of society and economy with the environment.

The specific application in energy consumption and $\mathrm{CO}_{2}$ emissions is the so called Kaya identity [4]. The Kaya identity is a linking expression of factors that determine the level of human impact on environment, in the form of $\mathrm{CO}_{2}$ emissions. It states that total emission level can be expressed as the

\footnotetext{
${ }^{1}$ Human Impact (I) on the environment equals the product of $\mathrm{P}=$ Population, $\mathrm{A}=$ Affluence, $\mathrm{T}=\mathrm{Technology}$. This describes how our growing population, affluence, and technology contribute toward our environmental impact.
}

product of four inputs: population, GDP per capita, energy use per unit of GDP, carbon emissions per unit of energy consumed. The Kaya identity ${ }^{2}$ plays a core role in the development of future emissions scenarios in the IPCC Special Report on Emissions Scenarios [5]. The scenarios set out a range of assumed conditions for future development of each of the four inputs. Population growth projections are available independently from demographic research; GDP per capita trends are available from economic statistics and econometrics; similarly to energy intensity and emission levels. The projected carbon emissions can drive carbon cycle and climate models to predict future $\mathrm{CO}_{2}$ concentration and climate change.

Some similar conceptual bases can be found in the field of decomposition analysis (DA). In particular, with the advent of the global oil crisis in 1973 and 1974, special attention was given to the use of energy in industry among policymakers because energy in

\footnotetext{
${ }^{2}$ Note that, a limitation of this equation is that it does not account for i) the direct release of carbon dioxide by deforestation through burning ii) the loss of the carbon sink due to that deforestation.
} 
industrial constituted most of the primary energy demand in most countries. Therefore, researchers focused on the mechanisms of change in industrial energy use. This new area of research emerged to quantify the impact of a structural change in industrial production on the total energy demand. These initial studies showed a significant impact of structural changes on the trends of energy demand. The need to identify and quantify its impact became an imperative for policy-making. This line of research was expanded considerably in the methodology and in its application; it is now a widely accepted tool for the formulation of national policies on energy and environment analysis [6]. It is particularly useful to provide the analysis of contributing factors, such as structural changes and changes in energy intensity. Steenhof et al. (2006) [7] manifested that decomposition of a predefined set of factors helps to understand the progression of the driving forces, the consequences of the processes occurring and the political dimensions associated with these processes. The same author also proposed that this would allow a rationalisation for possible progression into the future [7].

The scope of the IDA was expanded beyond the analysis of industrial energy demand, now being used in the analysis, at country level, of fields such as energy or environment ${ }^{3}$.

The need for political views ${ }^{4}$ of the IDA has mainly focused on historical analysis of the driving forces. While decomposition techniques such as IPAT can be used to predict future changes in the driving forces of a given system [8], DA is on the cusp of a new scenario analysis techniques and forecasting. For defining areas of future research in DA, Ang and Zhang (2000) [9] suggest its use in projecting energy demand and emissions in short and medium term. Sun (2001) [10] used a complete decomposition method to forecast GHG emissions in the EU-15 up to 2010. Sorrell et al. (2009) [11] recommended more research in the use of the decomposition framework for scenario development. Although both, IDA in energy and emissions, as well as scenario analysis in the context of energy emissions are often based on the framework of Kaya, the combination of these approaches has often not applied. In this line some studies have combined these approaches [7], [12]-[14].

This work discusses the DA methodology and is applied within Ecuadorian case. We present the used technique, the applied mathematical methodology and the construction of an appropriate identity to measure

\footnotetext{
${ }^{3}$ Energy efficiency measures are required by several international and national policies as the EU directive 2006/32/EC and while these can be executed using tools like DA and LMDI (Logarithmic Mean Divisia Index) techniques [6].

${ }^{4}$ The development of policy, reporting and monitoring of progress depends on the right as the index decomposition analysis analytical tools.
}

the change of $\mathrm{CO}_{2}$ emission in Ecuador during the period 1980-2025.

\section{MATERIALS AND METHODS \\ A. Logarithmic Mean Divisia Index}

As it was already explained, Ang (2004) [6] compared various index decomposition analysis methods and concluded that the multiplicative and additive Logarithmic Mean Divisia Index (LMDI) method is the preferred method due to their its theoretical foundation, adaptability, ease of use and result interpretation, and some other desirable properties in the context of decomposition analysis.

The used approach is a variation of the Kaya identity, where the amount of $\mathrm{CO}_{2}$ emissions from industry and from other energy uses may be studied quantifying the contributions of five different factors: i) global industrial activity, ii) industry activity mix, iii) sectorial energy intensity, iv) sectorial energy mix and v) $\mathrm{CO}_{2}$ emission factors. Moreover, we consider different sub-categories concerning the industrial sectors and the fuel type. The $\mathrm{CO}_{2}$ emissions can be written as,

$$
C=\sum_{i j} C_{i j}=\sum_{i j} Q \cdot S_{i} \cdot E I_{i} \cdot M_{i j} \cdot U_{i j},
$$

where $C$ is the total $\mathrm{CO}_{2}$ emissions (in a given year); $C i j$ is the $\mathrm{CO}_{2}$ emission arising from fuel type $j$ in the productive sector $i$; $\mathrm{Q}$ is the total GDP of the country; $S i(Q i / Q)$ is the share of sector $i$ in the total GDP; the energy intensity of sector $i$ is given by EIi (Ei/Qi); the energy matrix is given by $M i j(E i j / E i)$ and the $\mathrm{CO}_{2}$ emission factor by $U i j(C i j / E i j)$.

Throughout this work, as a convention, we will always refer to the productive sector with the $i$ index and to the type of energy source with the $j$ index.

This equation is an extension of the Kaya identity because we disaggregate in type of productive sector and kind of fuel used, while in the original formulation only aggregated terms are considered: $C$, $Q$, and $E$.

In this section we will present the methodology that has been applied based on the LMDI approach [15]. This analysis allows us to determine the relative importance of each term conforming the $\mathrm{CO}_{2}$ emission (see Equation 3). Indeed, it is very enlightening to write down the increase of $\mathrm{CO}_{2}$ emission relative to the value of a given period, and to decompose it as the sum or product of the terms corresponding to the different driving forces that conform the $\mathrm{CO}_{2}$ emission. i) In the case of the additive decomposition:

$$
\begin{gathered}
\Delta C \text { tot }=C^{T}-C^{0}, \\
\Delta C \text { tot }=\Delta C a c t+\Delta C s t r+\Delta C \operatorname{Cint}+\Delta C \operatorname{mix}+\Delta C e m f,
\end{gathered}
$$

where $C$ tot is the $\mathrm{CO}_{2}$ emission (relative to the base year), $C^{0}$ and $C^{T}$ represent the emission in the base and final year respectively, Cact is the GDP term, 
Cstr is the structure term (the share of the different sectors to the GDP), Cint the energy intensity term, Cmix the energy mixing term, and Cemf the emission factor term. Note that because the emission factors, given by the IPCC, do not change over the time, Cemf $=0$ all the time and therefore it will not be shown in the tables. ii) In the case of the multiplicative decomposition:

$$
\text { Dtot }=D^{T}-D^{0},
$$

Dtot $=$ Dact $X$ Dstr $X$ Dint $X$ Dmix $X$ Demf,

where Dtot is the $\mathrm{CO}_{2}$ emission (relative to the base year), Dact is the GDP term, Dstr is the structure term (the share of the different sectors to the GDP), Dint the energy intensity term, Dmix the energy mixing term, and Demf the emission factor term. As said before $\operatorname{Demf}=1$ all the time and therefore it will not be shown in the tables.

Applying as indicated before for the case of $\mathrm{CO}_{2}$ emissions (see Equation 3 and 5) the following formulas are obtained for decomposing changes in each of the terms involved in Equation 1, for both additive and multiplicative forms:

LMDI formula additive decomposition is,

$$
\begin{gathered}
\Delta C a c t=\sum_{i j} \frac{C_{i j}^{T}-C_{i j}^{0}}{\ln C_{i j}^{T}-\ln C_{i j}^{0}}\left(\frac{Q^{T}}{Q^{0}}\right), \\
\Delta C s t r=\sum_{i j} \frac{C_{i j}^{T}-C_{i j}^{0}}{\ln C_{i j}^{T}-\ln C_{i j}^{0}}\left(\frac{S_{i}^{T}}{S_{i}^{0}}\right), \\
\Delta C \operatorname{int}=\sum_{i j} \frac{C_{i j}^{T}-C_{i j}^{0}}{\ln C_{i j}^{T}-\ln C_{i j}^{0}}\left(\frac{E I_{i}^{T}}{E_{i}^{0}}\right), \\
\Delta C m i x=\sum_{i j} \frac{C_{i j}^{T}-C_{i j}^{0}}{\ln C_{i j}^{T}-\ln C_{i j}^{0}}\left(\frac{M_{i j}^{T}}{M_{i j}^{0}}\right), \\
\Delta C e m f=\sum_{i j} \frac{C_{i j}^{T}-C_{i j}^{0}}{\ln C_{i j}^{T}-\ln c_{i j}^{0}}\left(\frac{U_{i j}^{T}}{U_{i j}^{0}}\right),
\end{gathered}
$$

LMDI formula multiplicative decomposition is,

$$
\begin{aligned}
& \text { Dact }=\exp \left(\sum_{i j} \frac{\left(c_{i j}^{T}-C_{i j}^{0}\right) /\left(\ln C_{i j}^{T}-\ln C_{i j}^{0}\right)}{\left(C^{T}-C^{0}\right) /\left(\ln C^{T}-\ln C^{0}\right)} \ln \left(\frac{Q^{T}}{Q^{0}}\right)\right), \\
& D s t r=\exp \left(\sum_{i j} \frac{\left(c_{i j}^{T}-C_{i j}^{0}\right) /\left(\ln C_{i j}^{T}-\ln C_{i j}^{0}\right)}{\left(C^{T}-C^{0}\right) /\left(\ln C^{T}-\ln C^{0}\right)} \ln \left(\frac{S_{i}^{T}}{S_{i}^{0}}\right)\right), \\
& \text { Dint }=\exp \left(\sum_{i j} \frac{\left(C_{i j}^{T}-C_{i j}^{0}\right) /\left(\ln C_{i j}^{T}-\ln C_{i j}^{0}\right)}{\left(C^{T}-C^{0}\right) /\left(\ln C^{T}-\ln C^{0}\right)} \ln \left(\frac{E I_{i}^{T}}{E I_{i}^{0}}\right)\right), \\
& \text { Dmix }=\exp \left(\sum_{i j} \frac{\left(C_{i j}^{T}-C_{i j}^{0}\right) /\left(\ln C_{i j}^{T}-\ln c_{i j}^{0}\right)}{\left(C^{T}-C^{0}\right) /\left(\ln C^{T}-\ln C^{0}\right)} \ln \left(\frac{M_{i j}^{T}}{M_{i j}^{0}}\right)\right), \\
& \text { Demf }=\exp \left(\sum_{i j} \frac{\left(c_{i j}^{T}-C_{i j}^{0}\right) /\left(\ln C_{i j}^{T}-\ln C_{i j}^{0}\right)}{\left(C^{T}-C^{0}\right) /\left(\ln C^{T}-\ln C^{0}\right)} \ln \left(\frac{U_{i j}^{T}}{U_{i j}^{0}}\right)\right),
\end{aligned}
$$

B. Proposal of scenarios for Ecuador 2010-2025

Taking into account the general purpose of improve the quality of life of people with the least environmental impact and specific goals on each case, we propose four scenarios concerning the growth of the income, the evolution of the energy matrix and of the productive sectorial structure for the period 2011-2025.

1) Baseline scenario $(B S)$ : the GDP, the energy matrix and the productive sectorial structure will evolve through the smooth trend of the period 19802010 extrapolated to 2011-2025 using the geometric growth rate method.

2) Increasing GDP scenario $(S C$-2): GDP will increase approximately up to be double of reference GDP (2010) by 2025 in order to by 2025 the GDP per capita will reach the international average according to our estimates based on World Bank data) through a process of industrialization and improvement of the productive sectorial structure of the country. To generate this scenario a constant annual growth of GDP formation components of 7\% per year between 2011 to 2025 will be assumed and a structural change in the productive sectorial structure will be implemented through a growth of $1 \%$ per year in the GDP share in the sectors with more profit in the country's economy (industry sector and trade and public service sector). The rest of the variables will evolve as in the $B S$ scenario. This scenario clearly corresponds to a situation where the economy is growing rapidly and no mitigation measurements to reduce the $\mathrm{CO}_{2}$ emissions are carried out.

3) Increasing GDP and share of renewable energies scenario $(S C-3)$ : increasing GDP and change in productive sectorial structure as in the $S C-2$ scenario is considered, however the share of fossil energy, will be reduced approximately by one point per year, passing from a $88 \%$ in 2011 to a $67 \%$ in 2025 due to a constant annual growth of share in renewable and alternative energy, so then, the use of renewable energy will be increased up to almost $30 \%$ of the total energy consumption. This scenario shows a first measure of environmental responsibility in order to try to reduce dependence of fossil energy.

4) Increasing GDP and share of renewable energies and improvement in energy efficiency scenario (SC-4): increasing GDP, change in productive sectorial structure and change in share of fossil energy as in $S C$-3 scenario is carried out. Moreover, an improvement in energy efficiency is implemented with a $1 \%$ reduction of energy intensity in industry sector, in trade and public services sector and in transportation sector, so then; the energy efficiency will be enlarged by a reduction of the energy intensity and by changes in the productive sectorial structure. This scenario takes a step towards improving the country's environmental responsibility and sustainable development by supporting their energetic saving measures and energy efficiency.

Both $S C$ - 3 and $S C-4$ scenarios goals are realistic considering the state of development and evolution of energy technology in various energy projects implemented by the Ecuadorian government, and the trends in the use of renewable energies in the country [16]. 


\section{RESULTS AND DISCUSSION}

We will use three periods of 16 years to perform the analysis, two within the set of historical data (1980-1995 and 1995-2010) and the last one corresponding to the forecast period (2010-2025). This analysis will allow us to determine the relative importance of each term related to $\mathrm{CO}_{2}$ emission. The aggregate $\mathrm{CO}_{2}$ emissions in kilo tonnes of $\mathrm{CO}_{2}(\mathrm{kt})$, income in billions of USD (BUSD) and energy consumption in kilotonnes of oil equivalent (ktoe) are shown in Table I.

Table I.

\begin{tabular}{|c|c|c|c|}
\hline Year & $\begin{array}{c}\mathrm{CO}_{2} \\
\text { emissions (kt) }\end{array}$ & $\begin{array}{l}\text { Income } \\
\text { (BUSD) }\end{array}$ & $\begin{array}{c}\text { Energy } \\
\text { consumption } \\
\text { (ktoe) }\end{array}$ \\
\hline Data 1980 & 11,900 & 45,4 & 5032 \\
\hline Data 1995 & 19,600 & 63,4 & 7143 \\
\hline Data 2010 & 28,100 & 104 & 11930 \\
\hline BS 2025 & 55,000 & 167 & 20520 \\
\hline SC-2 2025 & 96,600 & 271 & 36040 \\
\hline SC-3 2025 & $66 ., 500$ & 244 & 32430 \\
\hline SC-3 2025 & 54,700 & 251 & 26700 \\
\hline
\end{tabular}

For The findings (see Fig. 1 and Fig. 2) show that in the period 1980-1995 there was an increase in emissions by $35 \%$ (see Table III) or equivalently of more than $5400 \mathrm{kt}$ (see Table II).

Table II.

Results of the $\mathrm{CO}_{2}$ emission additive decomposition factors for the period 1980-2025.

\begin{tabular}{c|ccccc} 
& \multicolumn{5}{|c}{ period 1980-2025. } \\
Scenario & $\Delta$ Ctot & $\Delta$ Cact & $\Delta$ Csrt & $\Delta$ Cint & $\Delta$ Cmix \\
\hline Data & 5410 & 4780 & 194 & 196 & 292 \\
$1980-1995$ & & & & & \\
Data & 14800 & 12300 & -637 & 1160 & 1990 \\
$1980-1995$ & & & & & \\
BS & 22600 & 20100 & -82 & 2500 & 96 \\
2025 & & & & & \\
SC-2 & 62100 & 55600 & 3020 & 3300 & 192 \\
2025 & 32500 & 39900 & 2400 & 2640 & -12400 \\
$\begin{array}{c}\text { SC-3 } \\
2025\end{array}$ & & & & & \\
SC-3 & 23200 & 37100 & 2200 & -5040 & -11100 \\
2025 & & & & &
\end{tabular}

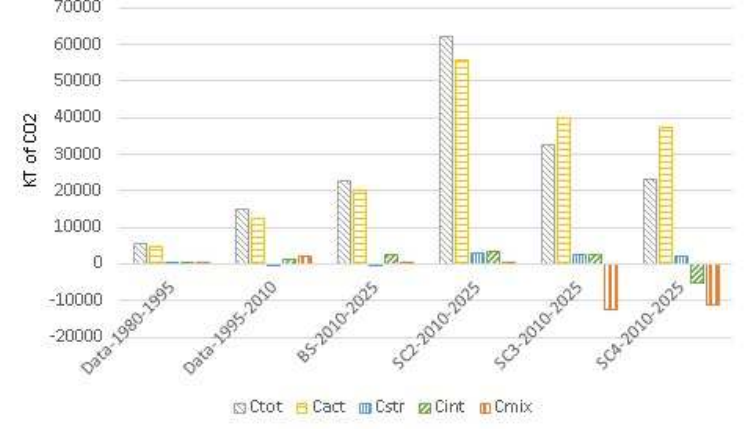

Fig. 1. Bar view of the $\mathrm{CO}_{2}$ emission additive decomposition factors for the period 1980-2025 in Ecuador.

The LMDI analysis shows that the activity effect led to an increased just 3 percent points (38\%), so that, the margin in emission increased. The effect of structural change $(D s t r=1: 01)$ in productive sectors and change in energy mix $($ Dmix $=1: 02)$ does not have significant impact over the emission in this period. Actual growth in emissions was lower because of the reduction of the sectorial energy intensity $($ Dint $=0: 95)$, see a pictorial view in Fig. 3 . Note that the ratio Dtot $=$ Dact is almost 1 and is a proxy of that country emissions in this period growth in the same factor that the income (see Fig. 1).

The period 1995-2010 reflected a greater increase in emissions $(85 \%)$ or equivalently of more than $14800 \mathrm{kt}$ (sees Tables II and III). The LMDI analysis shows that the activity effect led to an increase of $0: 80$ times $(68 \%)$, so that, the margin in emissions increase. In addition, changes in energy intensity $($ Dint $=1: 04)$ and in energy mix (Dint $=1: 09)$ led to an additional increased in emissions. The impact of structural change (Dstr $=0: 98)$ in productive sectors has a reduction effect in emission. Note that the ratio Dtot $=$ Dact equal to $1: 10$ is a proxy of the higher economic growth in this period (regarding to the previous one) accelerated the emission growth of the country (see Figures 3).

Table III.

Results of the $\mathrm{CO}_{2}$ emission multiplicative decomposition factors for the period 1980-2025.

\begin{tabular}{c|ccccc} 
Scenario & Dtot & Dact & Dsrt & Dint & Dmix \\
\hline Data & 1.35 & 1.38 & 1.01 & 0.95 & 1.02 \\
$1980-1995$ & & & & & \\
Data & 1.85 & 1.68 & 0.98 & 1.04 & 1.09 \\
$1980-1995$ & & & & & \\
BS & 1.72 & 1.61 & 1.00 & 1.07 & 1.00 \\
2025 & & & & & 1.00 \\
SC-2 & 3.03 & 2.59 & 1.09 & 1.07 & 1.00 \\
2025 & & & & & 0.77 \\
SC-3 & 2.08 & 2.33 & 1.09 & 1.07 & \\
2025 & & & & & 0.85 \\
SC-3 & 1.71 & 2.41 & 1.09 & &
\end{tabular}

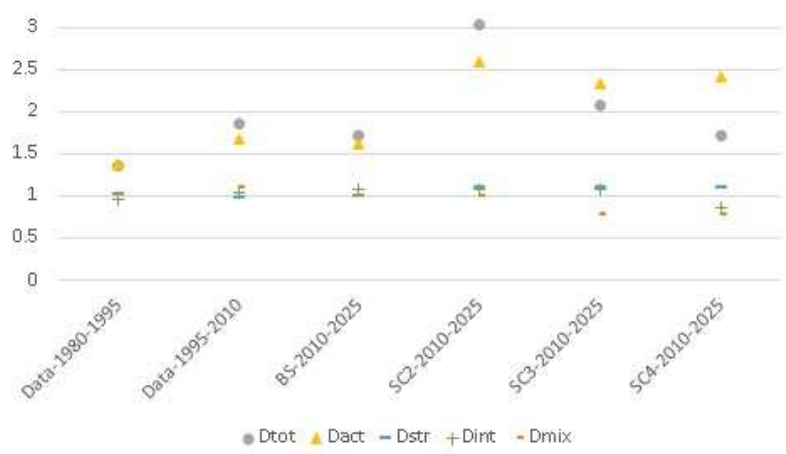

Fig. 2. View of the $\mathrm{CO}_{2}$ emission multiplicative decomposition factors for the period 1980-2025 in Ecuador.

Regarding to the forecast period, the findings show that in 2025 the $\mathrm{CO}_{2}$ emissions would increase by $72 \%$ or equivalently of more than $22000 \mathrm{kt}$ in the BS scenario. The LMDI analysis shows that the activity effect led to an increase of 0:85 times (61\%) that the margin in emissions increases. The effect of structural change $(D s t r=1: 00)$ in productive sectors and change in energy mix $($ Dmix $=1: 00)$ does not have impact on the emission in this period. Actual growth in emissions was higher because of the 
increase in sectorial energy intensity (Dint $=1: 07)$, as an pictorial view of Fig. 3. Note that the ratio Dtot $=$ Dact is almost the same as the previous period (1:07) (see Fig. 4) and is a proxy of the growth in emissions depends mainly on the scale term (Dact) in $B S$ scenario.

The SC-2 scenario presents an amount of emissions in 2025 that is more than 3 times in 2010 (3:03 times) or equivalently of more than $62000 \mathrm{kt}$. The LMDI analysis shows that the activity effect led to an increase of 0:57 times (2:49 times) so the margin in emissions increased. The effect of energy mix $($ Dmix $=1: 00)$ does not have impact on the emission during this period. As in BS scenario, actual growth in emissions was higher because of the increase in sectorial energy intensity $($ Dint $=1: 07)$ and by the impact of the structural change (Dstr $=$ 1:09), as in the pictorial view of Fig. 3. Note that the ratio Dtot $=$ Dact is 1:17 (higher than $B S$ scenario) (see Fig. 4) and is a proxy of the higher economic growth achieved in this scenario is because an increase in the economic scale and in energy intensity, arising from the shift in the composition of industry output towards energy-intensive sectors of the country as has been considered in this scenario.

The SC-3 scenario presents an amount of emissions in 2025 that is more than 2 times in 2010 (2:08 times) or equivalently of more than $32000 \mathrm{kt}$. The LMDI analysis shows that the activity effect led to an increase of 1:23 times (Dtot $=2: 33$ ) so the margin in emissions increased. In addition, impact of structural change $(D s t r=1: 09)$ in productive sectors changes and in energy intensity (Dint $=1: 07)$ led to an increase in emissions. The impact of energy mix $(D s t r=0: 77)$ used in productive sectors has a reduction effect in emissions as it has been considered in this scenario, see a pictorial view in Fig. 3. Note that the ratio Dtot $=$ Dact is lower than 1 $(0: 89)$ (see Fig. 4) and is a proxy for first time in the country (in the analyzed period), the growth of economics is higher than the growth of emissions. The reason is that in addition to the growth in the economic scale, the impact of energy mix change leads to a reduction of this ratio.

Finally, in the $S C-4$ scenario the emissions just increase by a factor of 1:71 or equivalently more than $23000 \mathrm{kt}$. The LMDI analysis shows that the activity effect led to an increase almost 2 times (Dtot $=2: 33$ ) the margin in emission increased. As in previous scenarios, the impact of structural change (Dstr = 1:09) in productive sectors changes led to an increased in emissions. However, actual growth in emissions was lower than in rest of scenarios because the impact of energy mix $(D s t r=0: 77)$ and the reduction in sectorial energy intensity (Dint $=0: 85$ ) has a reduction effect in emission as it has been considered in this scenario, see a pictorial view in Fig. 3. Note that in this scenario the ratio Dtot $=$ Dact is the lowest (0:71) (see Fig. 4) and as in the $S C-3$ scenario, in addition to the growth in the economic scale, the impact energy mix are present and adding the impact of the reduction of energy intensity considered in this scenario is reducing even more this ratio.

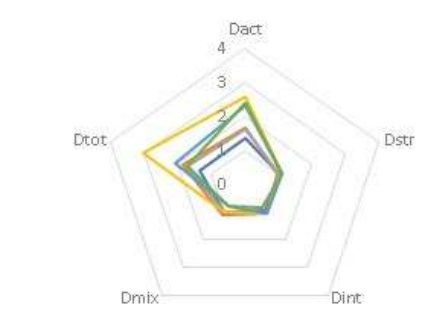

—Data-1980-1995 —Datr-1995-2010 — BS-2010-2025

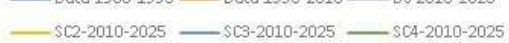

Fig. 3. Pictorial view of the $\mathrm{CO}_{2}$ emission multiplicative decomposition factors for the period 1980-2025 in Ecuador.

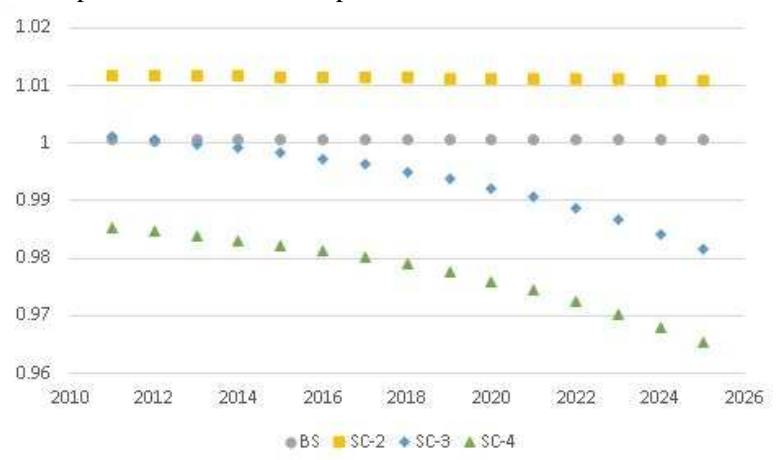

Fig. 4. $\quad$ Dtot $=$ Dact for the period 2011-2025 in Ecuador.

All the coefficients are summarized in Table II and III and in a pictorial way in Fig. 3. In this figure five axes are depicted corresponding to the five columns appearing in table III. The value of the vertical axis, Dtot, corresponds to the product of the five remaining variables, Dact, Dstr, Dint, Dmix and Demf.

\section{CONCLUSION}

This work presents a decomposition analysis of $\mathrm{CO}_{2}$ related to income growth and energy consumption bases on Logarithmic Mean Divisia Index (LMDI) analysis for Ecuador in the period of 1980-2025. For this purpose three periods have been selected, the first sub-period is 1980-1995 where the LMDI analysis findings concluded that the country emissions in this period almost grow (38\%) in the same factor that the income $(35 \%)$, see Fig. 1 . The second sub-period is 1995-2010 and the evidence concluded that a higher economic growth $(68 \%)$ led to an even greater emissions growth $(85 \%)$ in the country.

The third sub-period is 2010-2025 and it includes the analysis for the different macro-scenarios that have been proposed. To see more clearly how the income- $\mathrm{CO}_{2}$ relationship behaves as a function of time, it is very enlightening to depict the ratio Dtot $=$ 
Dact as a function of the time (see Fig. 4). The first striking thing is the very different behaviour for each scenario. On one hand, it is somehow surprising the almost flat curve corresponding to the $B S$ scenario which implies a trend-growth GDP scenario; however the $\mathrm{CO}_{2}$ emission increases steadily because of the absence of attenuation measurements. A similar behaviour, although slightly sloping down, is observed for $S C$-2, where a rapid growth of the GDP is assumed without any attenuation action regarding $\mathrm{CO}_{2}$ emission. It is worth noting a certain decrease of the ratio Dtot $=$ Dact in the final part of the period under this study. The other two scenarios, $S C-3$ and $S C-4$, show a steady reduction of the ratio Dtot $=$ Dact due to the changes in the sectorial structure and in the energy mix, which allows a compensation of rapid GDP growth.

This study combines decomposition analysis with a basic scenario modelling to create a baseline prevision as guidance for possible new policies. This allowed the development of an approach with a set of integrated exploratory scenarios about income growth, energy use and $\mathrm{CO}_{2}$ emissions for Ecuador in a medium term (2025). The scenarios show plausible more environmental-friendly pathways that the country could take to get closer to a sustainable development.

This preliminary analysis suggests that, with the appropriate changes in the energy mix, the sectorial structure, and the share of renewable energies, Ecuador can move into a more environmentally sustainable state. All these results encourage us to perform a more rigorous analysis and macroscenarios in regard to income and emission relationship.

\section{V.ACKNOWLEDGMENTS}

This study was partly supported by the Escuela Politécnica Nacional (EPN) of Ecuador. Research Project No PIS-16-02.

\section{REFERENCES}

[1] R. York, E. A. Rosa, and T. Dietz, "STIRPAT, IPAT and ImPACT: analytic tools for unpacking the driving forces of environmental impacts," Ecological Economics 46(3), 351365, 2003.

[2] B. Commoner, "A Bulletin Dialogue on The Closing Circle," Response. Bulletin of the Atomic Scientists 28 (17), 42-56, 1972.

[3] P. R. Ehrlich and J. Holdren, "A Bulletin Dialogue on the Closing Circle: Critique. One-Dimensional Ecology," Bulletin of the Atomic Scientists 28:16-27, 1972.

[4] Y. Kaya, "Impact of Carbon Dioxide Emission Control on GNP Growth: Interpretation of Proposed Scenarios," Paper presented to the IPCC Energy and Industry Subgroup, Response Strategies Working Group, Paris, (mimeo), 1990.

[5] IPCC, IPCC Special Reports. Emissions Scenarios. Cambridge University Press, The Edinburgh Building Shaftesbury Road, Cambridge CB22RU England, 2000.

[6] B. Ang, "Decomposition analysis for policymaking in energy: which is the preferred method?" Energy Policy 32, 2004.

[7] P. Steenhof, C. Woudsma, and E. Sparling, Greenhouse gas emissions and the surface transport of freight in Canada. Transportation Research Part D-Transport and Environment 11 (5), 369-376, 2006.

[8] P. Waggoner and J. Ausubel, "A framework for sustainability science: a renovated IPAT identity," Proceedings of the National Academy of Sciences 99 (12), 7860-7865, 2002.

[9] B. Ang and F. Zhang, "A survey of index decomposition analysis in energy and environmental analysis," Energy 25, 1149-1176, 2000.

[10] J. Sun, "Energy demand in the fifteen European Union countries by 2010 -A forecasting model based on the decomposition approach," Energy 26, 549-560, 2001.

[11] S. Sorrell, M. Lehtonen, L. Stapleton, J. Pujol, and T. Champion, "Decomposing road freight energy use in the United Kingdom,” Energy Policy 37 (8), 3115-3129, 2009.

[12] T. Kwon, "A scenario analysis of $\mathrm{CO} 2$ emission trends from car travel: Great Britain 20002030," Transport Policy 12 (2), 175-184, 2005.

[13] P. Steenhof, "Decomposition for emission baseline setting in China's electricity sector," Energy Policy 35 (1), 280294, 2007

[14] P. Agnolucci, G. Ekins, P. abd Iacopini, K. Anderson, A. Bows, S. Mander, and S. Shackley, "Different scenarios for achieving radical reduction in carbon emissions: a decomposition analysis," Ecological Economics 68, 16521666, 2009.

[15] B. Ang, "The LMDI approach to decomposition analysis: a practical guide," Energy Policy 33 (2005) 867871, 2005.

[16] A. Mosquera-Rodr'1guez, Policies and Strategies for Changing the Energy Matrix in Ecuador. Ministry of Electricity and Renewable Energy, Ecuador, 2008. 